\title{
Improved Results on Robust Stability for Systems with Interval Time-Varying Delays and Nonlinear Perturbations
}

\author{
Xin Zhou, Hexin Zhang, Xiaoxiang Hu, Junjun Hui, and Tianmei Li \\ Department of Automation, Xian Research Institute of Hi-Tech, Hongqing Town, Xi'an 710025, China \\ Correspondence should be addressed to Xin Zhou; ascentor@163.com
}

Received 4 May 2014; Accepted 10 June 2014; Published 30 June 2014

Academic Editor: Ligang Wu

Copyright ( 2014 Xin Zhou et al. This is an open access article distributed under the Creative Commons Attribution License, which permits unrestricted use, distribution, and reproduction in any medium, provided the original work is properly cited.

\begin{abstract}
This paper investigated delay-dependent robust stability criteria for systems with interval time-varying delays and nonlinear perturbations. A delay-partitioning approach is used in this paper, the delay-interval is partitioned into multiple equidistant subintervals, a new Lyapunov-Krasovskii (L-K) functional contains some triple-integral terms, and augment terms are introduced on these intervals. Then, by using integral inequalities method together with free-weighting matrix approach, a new less conservative delay-dependent stability criterion is formulated in terms of linear matrix inequalities (LMIs), which can be easily solved by optimization algorithms. Numerical examples are given to show the effectiveness and the benefits of the proposed method.
\end{abstract}

\section{Introduction}

Time-delay phenomena are ubiquitous in many practical systems such as communication systems, nuclear reactors, aircraft stabilization, and process control systems, which are often major sources of instability and poor performance. Hence, stability analysis and stabilization of systems with time-delays have received considerable attention in the past few decades [1-14]. Recently, many researchers pay attention to the interval time-varying delay, wherein the delay varies in a range for which the lower bound is not restricted to be zero. A typical example with interval time delay is the network control systems [2].

The basic framework for stability analysis and synthesis of stabilizing controllers is L-K functional and linear matrix inequality (LMI). Under this framework, an important issue was to enlarge the feasible region of stability criteria. To derive the delay-dependent stability conditions, many methods have been reported in the literature. For example, the free-weighting matrix method was used in [36]; Jensen's integral inequality method was adopted in [713]. Recently, inspired by the discretized Lyapunov method, delay-partitioning approach was proposed in $[14,15]$, wherein the delay-interval was uniformly divided into multiple segments, choosing proper functional with different weighted matrices corresponding to different segments. A new technique called delay-central point method was proposed in [16]. Based on the delay-central point method and decomposition technique, [17] proposed a less conservative stability criterion for computing the maximum allowable bound of the delay range. As an extension of delay-central point method, a new delay-partitioning approach was proposed in [18] for the uncertain stochastic systems with interval time-varying delay. Referring to the nonlinearities, as time delays, also can cause instability and poor performance of practical systems. Therefore, the stability problem of time-delay systems with nonlinear perturbations has received increasing attention [19-24]. A descriptor model transformation was employed in [19]. The free-weighting matrices approach was adopt in [20, 21]. Recently, a less conservative delay-dependent stability criterion was provided in [22] by partitioning the delayinterval into two segments of equal length and evaluating the time-derivative of a candidate $\mathrm{L}-\mathrm{K}$ functional in each segment of the delay-interval. The main advantage of the method [22] is that more information on the variation interval of the delay is employed, but we can employ information of timedelay much more if we partition the delay-interval into more segments.

Inspired by the idea of $[18,22]$, in this paper, we divide the variation interval of the delay into $N$ parts with equal 
length and construct a new L-K functional with tripleintegral terms and augment terms for this delay-interval. Based on integral inequalities method together with freeweighting matrix approach, a new delay-dependent stability criterion for the system is formulated in terms of linear matrix inequalities, which can be easily calculated by using MATLAB LMI control toolbox. Numerical examples are given to illustrate the effectiveness and less conservatism of the proposed method.

\section{Problem Description and Preliminaries}

Consider the following neutral system with mixed timevarying delays and nonlinear perturbations:

$$
\begin{gathered}
\dot{x}(t)=\mathbf{A} x(t)+\mathbf{B} x(t-h(t)) \\
+f_{1}(x(t), t)+f_{2}(x(t-h(t)), t), \\
x(\theta)=\phi(\theta), \quad \dot{x}(\theta)=\varphi(\theta), \\
\forall \theta \in\left[-\max \left(h_{M}, \tau\right), 0\right],
\end{gathered}
$$

where $x(t) \in \mathbb{R}^{n}$ is the state vector, $\mathbf{A}$ and $\mathbf{B}$ are constant matrices with appropriate dimensions, and $h(t)$ is a timevarying delay satisfying

$$
0 \leq h_{m} \leq h(t) \leq h_{M}, \quad \dot{h}(t) \leq \mu, \quad \forall t \geq 0,
$$

where $h_{m}$ and $h_{M}$ represent the lower and upper bounds of the time-varying delay $h(t)$, respectively, $\mu$ is the bound on the delay-derivative, and initial condition $\varphi(t)$ is a continuous vector-valued function. The functions $f_{1}(x(t), t)$ and $f_{2}(x(t-$ $h(t)), t$ ) are unknown nonlinear perturbations with respect to the current state $x(t)$ and in the delay state $x(t-h(t))$, respectively. They satisfy $f_{1}(0, t)=0, f_{2}(0, t)=0$, and

$$
\begin{gathered}
f_{1}^{T}(x(t), t) f(x(t), t) \leq \alpha^{2} x^{T}(t) x(t), \\
f_{2}^{T}(x(t-h(t)), t) f_{2}(x(t-h(t)), t) \\
\quad \leq \beta^{2} x^{T}(t-h(t)) x(t-h(t)),
\end{gathered}
$$

where $\alpha \geq 0, \beta \geq 0$ are given constants; for simplicity we denote $f_{1}=f_{1}(x(t), t), f_{2}=f_{2}(x(t-h(t)), t)$.

Before moving on, we need the following instrumental lemma.

Lemma 1 (see [10]). For any scalar $h(t) \geq 0$ and constant matrix $\mathbf{Q} \in \mathbb{R}^{n \times n}, \mathbf{Q}=\mathbf{Q}^{T}>0$, the following inequality holds:

$$
\begin{aligned}
-\int_{t-h(t)}^{t} \dot{x}^{T}(s) \mathbf{Q} \dot{x}(s) d s \leq & h(t) \zeta^{T}(t) \mathbf{V} \mathbf{Q}^{-1} \mathbf{V}^{T} \zeta(t) \\
& +2 \zeta^{T}(t) \mathbf{V}[x(t)-x(t-h(t))]
\end{aligned}
$$

where

$$
\begin{aligned}
\zeta^{T}(t)= & {\left[\begin{array}{llll}
x^{T}(t) & x^{T}\left(t-h_{i}\right) & x^{T}(t-h(t)) & x^{T}\left(t-h_{i+1}\right)
\end{array}\right.} \\
& \left.\dot{x}^{T}(t) \int_{t-h_{i}}^{t} x^{T}(s) d s \int_{t-h_{i+1}}^{t-h_{i}} x^{T}(s) d s \quad f_{1}^{T} f_{2}^{T}\right] .
\end{aligned}
$$

$\mathbf{V}$ is a free-weighting matrix with appropriate dimensions.

Lemma 2 (see [9]). For any constant matrix $\mathbf{W} \in \mathbb{R}^{n \times n}, \mathbf{W}=$ $\mathbf{W}^{T}>0$, a scalar function $h:=h(t)>0$ and a vector-valued function $\dot{x}:[-h, 0] \rightarrow R^{n}$, such that the following integrations are well defined; then

$$
\begin{gathered}
-h \int_{t-h}^{t} \dot{x}^{T}(s) \mathbf{W} \dot{x}(s) d s \leq \zeta_{1}^{T}(t)\left[\begin{array}{cc}
-\mathbf{W} & \mathbf{W} \\
\mathbf{W} & -\mathbf{W}
\end{array}\right] \zeta_{1}(t), \\
-\frac{h^{2}}{2} \int_{-h}^{0} \int_{t+\theta}^{t} \dot{x}^{T}(t) \mathbf{W} \dot{x}(t) d s \leq \zeta_{2}^{T}(t)\left[\begin{array}{cc}
-\mathbf{W} & \mathbf{W} \\
\mathbf{W} & -\mathbf{W}
\end{array}\right] \zeta_{2}(t)
\end{gathered}
$$

with $\zeta_{1}^{T}(t)=\left[x^{T}(t) x^{T}(t-h)\right]$ and $\zeta_{2}^{T}(t)=$ $\left[h x^{T}(t) \int_{t-h}^{t} x^{T}(s) d s\right]$.

Lemma 3 (see [25]). Suppose $\gamma_{1} \leq \gamma(t) \leq \gamma_{2}$, where $\gamma(\cdot)$ : $R_{+} \rightarrow R_{+}$. Then, for any constant matrices $\boldsymbol{\Xi}_{1}, \boldsymbol{\Xi}_{2}$, and $\mathbf{\Omega}$ with proper dimensions, the following matrix inequality

$$
\boldsymbol{\Omega}+\left(\gamma(t)-\gamma_{1}\right) \boldsymbol{\Xi}_{1}+\left(\gamma_{2}-\gamma(t)\right) \boldsymbol{\Xi}_{2}<0
$$

holds, if and only if

$$
\begin{aligned}
& \boldsymbol{\Omega}+\left(\gamma_{2}-\gamma_{1}\right) \boldsymbol{\Xi}_{1}<0, \\
& \boldsymbol{\Omega}+\left(\gamma_{2}-\gamma_{1}\right) \boldsymbol{\Xi}_{2}<0 .
\end{aligned}
$$

\section{Main Result}

In this section, we study the delay-dependent robust stability of system (1) based on the delay-partitioning approach.

Theorem 4. For given positive scalars $h_{m}, h_{M}, \mu, \alpha$, and $\beta$ system (1) with uncertainty (3) is asymptotically stable if there exist symmetric matrices

$$
\mathbf{P}=\left[\begin{array}{ccc}
\mathbf{P}_{11} & \mathbf{P}_{12} & \mathbf{P}_{13} \\
* & \mathbf{P}_{22} & \mathbf{P}_{23} \\
* & * & \mathbf{P}_{33}
\end{array}\right]
$$

and $\mathbf{Q}_{1}, \mathbf{Q}_{2}, \mathbf{Q}_{3}, \mathbf{Z}_{2}, \mathbf{Z}_{3}, \mathbf{R}_{2}, \mathbf{R}_{3}$, any matrices $\mathbf{K}_{a}, \mathbf{V}_{a}, \mathbf{W}_{a}(a=$ $1,2)$, and scalars $\varepsilon_{i} \geq 0(i=1,2)$ such that the following LMIs hold:

$$
\begin{aligned}
& {\left[\begin{array}{cc}
\Phi & \sqrt{h_{\delta}} \mathbf{W} \\
* & -\mathbf{Z}_{3}
\end{array}\right]<0, \quad i=1,2, \ldots, N,} \\
& {\left[\begin{array}{cc}
\Phi & \sqrt{h_{\delta}} \mathbf{V} \\
* & -\mathbf{Z}_{3}
\end{array}\right]<0, \quad i=1,2, \ldots, N,}
\end{aligned}
$$


where $\boldsymbol{\Phi}=\left(\boldsymbol{\Phi}_{i, j}\right)_{9 \times 9}$ with

$$
\begin{aligned}
& \boldsymbol{\Phi}_{11}=\mathbf{P}_{12}+\mathbf{P}_{12}^{T}+\mathbf{Q}_{1}+\mathbf{Q}_{2}+\mathbf{Q}_{3}-\mathbf{Z}_{2}-h_{i}^{2} \mathbf{R}_{2} \\
& -h_{\delta}^{2} \mathbf{R}_{3}+\mathbf{K}_{1} \mathbf{A}+\mathbf{A}^{T} \mathbf{K}_{1}^{T}+\varepsilon_{1} a^{2}, \\
& \boldsymbol{\Phi}_{12}=\mathbf{Z}_{2}-\mathbf{P}_{12}+\mathbf{P}_{13}, \quad \Phi_{13}=\mathbf{K}_{1} \mathbf{B}, \\
& \boldsymbol{\Phi}_{14}=-\mathbf{P}_{13}, \quad \boldsymbol{\Phi}_{15}=\mathbf{P}_{11}-\mathbf{K}_{1}+\mathbf{A}^{T} \mathbf{K}_{2}^{T}, \\
& \boldsymbol{\Phi}_{16}=\mathbf{P}_{22}^{T}+h_{i} \mathbf{R}_{2}, \quad \boldsymbol{\Phi}_{17}=\mathbf{P}_{32}^{T}+h_{\delta} \mathbf{R}_{3}, \\
& \boldsymbol{\Phi}_{18}=\boldsymbol{\Phi}_{19}=\mathbf{K}_{1}, \quad \boldsymbol{\Phi}_{22}=-\mathbf{Q}_{2}-\mathbf{Z}_{2}+\mathbf{V}_{1}+\mathbf{V}_{1}^{T}, \\
& \Phi_{23}=-\mathbf{V}_{1}+\mathbf{V}_{2}^{T}, \quad \Phi_{24}=\Phi_{25}=0, \\
& \boldsymbol{\Phi}_{26}=-\mathbf{P}_{22}^{T}+\mathbf{P}_{23}^{T}, \quad \boldsymbol{\Phi}_{27}=-\mathbf{P}_{32}^{T}+\mathbf{P}_{33}^{T} \text {, } \\
& \Phi_{28}=\Phi_{29}=0, \\
& \boldsymbol{\Phi}_{33}=-(1-\mu) \mathbf{Q}_{1}-\mathbf{V}_{2}-\mathbf{V}_{2}^{T}+\mathbf{W}_{1}+\mathbf{W}_{1}^{T}+\varepsilon_{2} \beta^{2}, \\
& \boldsymbol{\Phi}_{34}=-\mathbf{W}_{1}+\mathbf{W}_{2}^{T}, \quad \boldsymbol{\Phi}_{35}=\mathbf{B}^{T} \mathbf{K}_{2}^{T}, \\
& \boldsymbol{\Phi}_{36}=\cdots=\boldsymbol{\Phi}_{39}=0, \quad \Phi_{44}=-\mathbf{Q}_{3}-\mathbf{W}_{2}-\mathbf{W}_{2}^{T}, \\
& \boldsymbol{\Phi}_{45}=0, \quad \boldsymbol{\Phi}_{46}=-\mathbf{P}_{23}^{T}, \\
& \boldsymbol{\Phi}_{47}=-\mathbf{P}_{33}^{T}, \quad \boldsymbol{\Phi}_{48}=\boldsymbol{\Phi}_{49}=0, \\
& \boldsymbol{\Phi}_{55}=h_{i}^{2} \mathbf{Z}_{2}+h_{\delta} \mathbf{Z}_{3}+\frac{1}{4} h_{i}^{4} \mathbf{R}_{2} \\
& +\frac{1}{4}\left(h_{i+1}^{2}-h_{i}^{2}\right)^{2} \mathbf{R}_{3}-\mathbf{K}_{2}-\mathbf{K}_{2}^{T}, \\
& \boldsymbol{\Phi}_{56}=\mathbf{P}_{21}^{T}, \quad \boldsymbol{\Phi}_{57}=\mathbf{P}_{31}^{T}, \\
& \boldsymbol{\Phi}_{58}=\boldsymbol{\Phi}_{5,9}=\mathbf{K}_{2}, \quad \boldsymbol{\Phi}_{66}=-\mathbf{R}_{2}, \\
& \Phi_{67}=\cdots=\Phi_{69}=0, \\
& \Phi_{77}=-\mathbf{R}_{3}, \quad \Phi_{78}=\Phi_{79}=0, \\
& \Phi_{88}=-\varepsilon_{1} \mathbf{I}, \quad \Phi_{89}=0, \quad \Phi_{99}=-\varepsilon_{2} \mathbf{I}, \\
& h_{\delta}=h_{i+1}-h_{i}=\frac{\left(h_{M}-h_{m}\right)}{N}, \\
& h_{i}=h_{1}+\frac{(i-1)\left(h_{M}-h_{m}\right)}{N}, \\
& \mathbf{K}=\left[\begin{array}{lllllllll}
\mathbf{K}_{1}^{T} & 0 & 0 & 0 & \mathbf{K}_{2}^{T} & 0 & 0 & 0 & 0
\end{array}\right]^{T} \text {, } \\
& \mathbf{V}=\left[\begin{array}{lllllllll}
0 & \mathbf{V}_{1}^{T} & \mathbf{V}_{2}^{T} & 0 & 0 & 0 & 0 & 0 & 0
\end{array}\right]^{T} \text {, } \\
& \mathbf{W}=\left[\begin{array}{lllllllll}
0 & 0 & \mathbf{W}_{1}^{T} & \mathbf{W}_{2}^{T} & 0 & 0 & 0 & 0 & 0
\end{array}\right]^{T} .
\end{aligned}
$$

Proof. First, we decompose the delay-interval $\left[h_{m}, h_{M}\right]$ into $N$ equidistant subinterval, where $N$ is a given integer; that is,

$$
\begin{aligned}
& {\left[h_{m}, h_{M}\right]} \\
& \quad=\left[h_{1}, h_{2}\right] \cup\left[h_{2}, h_{3}\right] \cup \cdots \cup\left[h_{i}, h_{i+1}\right] \cup \cdots \cup\left[h_{N}, h_{N+1}\right] .
\end{aligned}
$$

Then, the L-K functional corresponding to the timevariation $h(t) \in\left[h_{2}, h_{3}\right]$ is chosen as

$$
\begin{aligned}
V_{2}(t)= & \xi^{T}(t) \mathbf{P} \xi(t)+\int_{t-h(t)}^{t} x^{T}(s) \mathbf{Q}_{1} x(s) d s \\
& +\int_{t-h_{2}}^{t} x^{T}(s) \mathbf{Q}_{2} x(s) d s+\int_{t-h_{3}}^{t} x^{T}(s) \mathbf{Q}_{3} x(s) d s \\
& +h_{2} \int_{-h_{2}}^{0} \int_{t+\theta}^{t} \dot{x}^{T}(s) \mathbf{Z}_{2} \dot{x}(s) d s d \theta \\
& +\int_{-h_{3}}^{-h_{2}} \int_{t+\theta}^{t} \dot{x}^{T}(s) \mathbf{Z}_{3} \dot{x}(s) d s d \theta \\
& +\frac{h_{2}^{2}}{2} \int_{-h_{2}}^{0} \int_{\theta}^{0} \int_{t+\lambda}^{t} \dot{x}^{T}(s) \mathbf{R}_{2} \dot{x}(s) d s d \lambda d \theta \\
& +\frac{\left(h_{3}^{2}-h_{2}^{2}\right)}{2} \int_{-h_{3}}^{-h_{2}} \int_{\theta}^{0} \int_{t+\lambda}^{t} \dot{x}^{T}(s) \mathbf{R}_{3} \dot{x}(s) d s d \lambda d \theta
\end{aligned}
$$

with $\xi^{T}(t)=\left[\begin{array}{lll}x^{T}(t) & \int_{t-h_{2}}^{t} x^{T}(t) d s & \int_{t-h_{3}}^{t-h_{2}} x^{T}(t) d s\end{array}\right]$.

The time-derivative of the $\mathrm{L}-\mathrm{K}$ functional along the trajectory of (1) is given by

$$
\begin{aligned}
\dot{V}_{2}(t)= & 2 \xi^{T}(t) \mathbf{P} \dot{\xi}(t)+x^{T}(t)\left(\mathbf{Q}_{1}+\mathbf{Q}_{2}+\mathbf{Q}_{3}\right) x(t) \\
& -(1-\mu) x^{T}(t-h(t)) \mathbf{Q}_{1} x(t-h(t)) \\
& -x^{T}\left(t-h_{2}\right) \mathbf{Q}_{2} x\left(t-h_{2}\right)-x^{T}\left(t-h_{3}\right) \mathbf{Q}_{3} x\left(t-h_{3}\right) \\
& +\dot{x}^{T}(t)\left[h_{2}^{2} \mathbf{Z}_{2}+\left(h_{3}-h_{2}\right) \mathbf{Z}_{3}\right] \dot{x}(t) \\
& -h_{2} \int_{t-h_{2}}^{t} \dot{x}^{T}(s) \mathbf{Z}_{2} \dot{x}(s) d s-\int_{t-h_{3}}^{t-h_{2}} \dot{x}^{T}(s) \mathbf{Z}_{3} \dot{x}(s) d s \\
& +\frac{1}{4} h_{2}^{4} \dot{x}^{T}(t) \mathbf{R}_{2} \dot{x}(t)+\frac{1}{4}\left(h_{3}^{2}-h_{2}^{2}\right)^{2} \dot{x}^{T}(t) \mathbf{R}_{3} \dot{x}(t) \\
& -\frac{h_{2}^{2}}{2} \int_{-h_{2}}^{0} \int_{t+\theta}^{t} \dot{x}^{T}(s) \mathbf{R}_{2} \dot{x}(t) d s d \theta \\
& -\frac{\left(h_{3}^{2}-h_{2}^{2}\right)}{2} \int_{-h_{3}}^{-h_{2}} \int_{t+\theta}^{t} \dot{x}^{T}(s) \mathbf{R}_{3} \dot{x}(t) d s d \theta .
\end{aligned}
$$


Note that

$$
\begin{aligned}
-\int_{t-h_{3}}^{t-h_{2}} \dot{x}^{T}(s) \mathbf{Z}_{3} \dot{x}(s) d s= & -\int_{t-h_{3}}^{t-h(t)} \dot{x}^{T}(s) \mathbf{Z}_{3} \dot{x}(s) d s \\
& -\int_{t-h(t)}^{t-h_{2}} \dot{x}^{T}(s) \mathbf{Z}_{3} \dot{x}(s) d s .
\end{aligned}
$$

From Lemmas 1 and 2, we have

$$
\begin{aligned}
& -\int_{t-h(t)}^{t-h_{2}} \dot{x}^{T}(s) \mathbf{Z}_{3} \dot{x}(s) d s \\
& \leq\left(h(t)-h_{2}\right) \zeta^{T}(t) \mathbf{V} \mathbf{Z}_{3}^{-1} \mathbf{V}^{T} \zeta(t) \\
& +2 \zeta^{T}(t) \mathbf{V}\left[x\left(t-h_{2}\right)-x(t-h(t))\right], \\
& -\int_{t-h_{3}}^{t-h(t)} \dot{x}^{T}(s) \mathbf{Z}_{3} \dot{x}(s) d s \\
& \leq\left(h_{3}-h(t)\right) \zeta^{T}(t) \mathbf{W} \mathbf{Z}_{3}^{-1} \mathbf{W}^{T} \zeta(t) \\
& +2 \zeta^{T}(t) \mathbf{W}\left[x(t-h(t))-x\left(t-h_{3}\right)\right] \\
& -h_{2} \int_{t-h_{2}}^{t} \dot{x}^{T}(s) \mathbf{Z}_{2} \dot{x}(s) d s \\
& \leq\left[\begin{array}{c}
x(t) \\
x\left(t-h_{2}\right)
\end{array}\right]^{T}\left[\begin{array}{cc}
-\mathbf{Z}_{2} & \mathbf{Z}_{2} \\
* & -\mathbf{Z}_{2}
\end{array}\right]\left[\begin{array}{c}
x(t) \\
x\left(t-h_{2}\right)
\end{array}\right], \\
& -\frac{h_{2}^{2}}{2} \int_{-h_{2}}^{0} \int_{t+\theta}^{t} \dot{x}^{T}(s) \mathbf{R}_{2} \dot{x}(t) d s d \theta \\
& \leq\left[\begin{array}{c}
h_{2} x(t) \\
\int_{t-h_{2}}^{t} x(s) d s
\end{array}\right]^{T}\left[\begin{array}{cc}
-\mathbf{R}_{2} & \mathbf{R}_{2} \\
\mathbf{R}_{2} & -\mathbf{R}_{2}
\end{array}\right]\left[\begin{array}{c}
h_{2} x(t) \\
\int_{t-h_{2}}^{t} x(s) d s
\end{array}\right], \\
& -\frac{\left(h_{3}^{2}-h_{2}^{2}\right)}{2} \int_{-h_{3}}^{-h_{2}} \int_{t+\theta}^{t} \dot{x}^{T}(s) \mathbf{R}_{3} \dot{x}(t) d s d \theta \\
& \leq\left[\begin{array}{c}
\left(h_{3}-h_{2}\right) x(t) \\
\int_{t-h_{3}}^{t-h_{2}} x(s) d s
\end{array}\right]\left[\begin{array}{cc}
-\mathbf{R}_{3} & \mathbf{R}_{3} \\
\mathbf{R}_{3} & -\mathbf{R}_{3}
\end{array}\right]\left[\begin{array}{c}
\left(h_{3}-h_{2}\right) x(t) \\
\int_{t-h_{3}}^{t-h_{2}} x(s) d s
\end{array}\right] .
\end{aligned}
$$

From (3), we can obtain, for any scalars $\varepsilon_{1}>0$, $\varepsilon_{2}>0$,

$$
\begin{aligned}
& \varepsilon_{1}\left[\alpha^{2} x^{T}(t) x(t)-f_{1}^{T}(x(t), t) f_{1}(x(t), t)\right] \geq 0, \\
& \varepsilon_{2}\left[\beta^{2} x^{T}(t-h(t)) x(t-h(t))\right. \\
& \left.\quad-f_{2}^{T}(x(t-h(t)), t) f_{2}(x(t-h(t)), t)\right] \geq 0 .
\end{aligned}
$$

From the system (1), we have the following equation:

$$
\begin{aligned}
& 2\left[x^{T}(t) \mathbf{K}_{1}+\dot{x}(t) \mathbf{K}_{2}\right] \\
& \quad \times[\mathbf{A} x(t)+\mathbf{B} x(t-h(t)) \\
& \left.\quad+f_{1}(x(t), t)+f_{2}(x(t-h(t)), t)-\dot{x}(t)\right]=0 .
\end{aligned}
$$

By substituting (17) (20) in (15) and defining an augmented state vector,

$$
\begin{aligned}
\zeta^{T}(t)= & {\left[\begin{array}{llll}
x^{T}(t) & x^{T}\left(t-h_{i}\right) & x^{T}(t-h(t)) & x^{T}\left(t-h_{i+1}\right)
\end{array}\right.} \\
& \left.\dot{x}^{T}(t) \quad \int_{t-h_{i+1}}^{t} x^{T}(s) d s \int_{t-h_{i+1}}^{t-h_{i}} x^{T}(s) d s \quad f_{1}^{T} f_{2}^{T}\right] .
\end{aligned}
$$

Then the time-derivative $\dot{V}_{2}(x(t), t)$ can be expressed as follows:

$$
\begin{aligned}
\dot{V}_{2}(x(t), t) \leq \zeta^{T}(t)(\boldsymbol{\Phi} & +\left(h_{3}-h(t)\right) \mathbf{W} \mathbf{Z}_{3}^{-1} \mathbf{W}^{T} \\
& \left.+\left(h(t)-h_{2}\right) \mathbf{V Z}_{3}^{-1} \mathbf{V}^{T}\right) \zeta(t) .
\end{aligned}
$$

One can see that if $h(t) \in\left[h_{2}, h_{3}\right]$,

$$
\Phi+\left(h_{3}-h(t)\right) \mathbf{W} \mathbf{Z}_{3}^{-1} \mathbf{W}^{T}+\left(h(t)-h_{2}\right) \mathbf{V Z}_{3}^{-1} \mathbf{V}^{T}<0 .
$$

Then $\dot{V}(t)<-\varepsilon\|x(t)\|^{2}$ for some scalar $\varepsilon>0$, from which we conclude that system (1) is asymptotically stable according to L-K stability theory [1].

Applying Lemma 3 to (23) yields the following inequality:

$$
\begin{gathered}
\boldsymbol{\Phi}+\left(h_{3}-h(t)\right) \mathbf{W Z}_{3}^{-1} \mathbf{W}^{T}<0, \\
\Phi+\left(h(t)-h_{2}\right) \mathbf{V Z}_{3}^{-1} \mathbf{V}^{T}<0 .
\end{gathered}
$$

By Schur complement on (24), we can obtain (10) and (11) with $i=2$.

Without loss of generality, when $h(t) \in\left[h_{i}, h_{i+1}\right](i=$ $1,3, \ldots, N)$, construct the following L-K functional:

$$
\begin{aligned}
V_{i}(t)= & \xi^{T}(t) \mathbf{P} \xi(t)+\int_{t-h(t)}^{t} x^{T}(s) \mathbf{Q}_{1} x(s) d s \\
& +\int_{t-h_{i}}^{t} x^{T}(s) \mathbf{Q}_{2} x(s) d s+\int_{t-h_{i+1}}^{t} x^{T}(s) \mathbf{Q}_{3} x(s) d s \\
& +h_{i} \int_{-h_{i}}^{0} \int_{t+\theta}^{t} \dot{x}^{T}(s) \mathbf{Z}_{2} \dot{x}(s) d s d \theta \\
& +\int_{-h_{i+1}}^{-h_{i}} \int_{t+\theta}^{t} \dot{x}^{T}(s) \mathbf{Z}_{3} \dot{x}(s) d s d \theta \\
& +\frac{h_{i}^{2}}{2} \int_{-h_{i}}^{0} \int_{\theta}^{0} \int_{t+\lambda}^{t} \dot{x}^{T}(s) \mathbf{R}_{2} \dot{x}(s) d s d \lambda d \theta \\
& +\frac{\left(h_{i+1}^{2}-h_{i}^{2}\right)}{2} \int_{-h_{i+1}}^{-h_{i}} \int_{\theta}^{0} \int_{t+\lambda}^{t} \dot{x}^{T}(s) \mathbf{R}_{3} \dot{x}(s) d s d \lambda d \theta
\end{aligned}
$$


with $\xi^{T}(t)=\left[x^{T}(t) \quad \int_{t-h_{i}}^{t} x^{T}(t) d s \quad \int_{t-h_{i+1}}^{t-h_{i}} x^{T}(t) d s\right]$, and $\mathbf{P}, \mathbf{Q}_{1}$, $\mathbf{Q}_{2}, \mathbf{Q}_{3}, \mathbf{Z}_{2}, \mathbf{Z}_{3}, \mathbf{R}_{2}, \mathbf{R}_{3}$ are the same matrix variables used in $V_{2}(t)$. Now, define an augmented state vector:

$$
\begin{aligned}
\zeta^{T}(t)= & {\left[\begin{array}{llll}
x^{T}(t) & x^{T}\left(t-h_{i}\right) & x^{T}(t-h(t)) & x^{T}\left(t-h_{i+1}\right)
\end{array}\right.} \\
& \left.\dot{x}^{T}(t) \int_{t-h_{i+1}}^{t} x^{T}(s) d s \int_{t-h_{i+1}}^{t-h_{i}} x^{T}(s) d s \quad f_{1}^{T} f_{2}^{T}\right] .
\end{aligned}
$$

Using the same method, we have the conditions (10) and (11) as that for $h(t) \in\left[h_{i}, h_{i+1}\right](i=1,3, \ldots, N)$. The proof is completed.

Remark 5. As an extension of the method used in $[18,22]$, we divide the delay-interval into $N$ subintervals, constructing a new L-K functional that contains some triple-integral terms and augment term for each delay-interval. This treatment makes us employ more information on the time delay and yields less conservative delay-range bounds.

Remark 6. If there is no perturbation, that is, $f=0, g=0$, then the stability problem of system (1) is reduced to analyze the stability of the system:

$$
\begin{aligned}
\dot{x}(t) & =\mathbf{A} x(t)+\mathbf{B} x(t-h(t)), \\
x(\theta) & =\phi(\theta), \quad \forall \theta \in\left[-h_{M}, 0\right] .
\end{aligned}
$$

According to Theorem 4, we can obtain the following corollary for the delay-dependent stability of system (27).

Corollary 7. For given positive scalars, $h_{m}, h_{M}, \mu$, system (27) with uncertainty (10) is asymptotically stable if there exist real symmetric positive definitive matrices

$$
\mathbf{P}=\left[\begin{array}{ccc}
\mathbf{P}_{11} & \mathbf{P}_{12} & \mathbf{P}_{13} \\
* & \mathbf{P}_{22} & \mathbf{P}_{23} \\
* & * & \mathbf{P}_{33}
\end{array}\right]
$$

and $\mathbf{Q}_{1}, \mathbf{Q}_{2}, \mathbf{Q}_{3}, \mathbf{Z}_{2}, \mathbf{Z}_{3}, \mathbf{R}_{2}, \mathbf{R}_{3}$, any matrices $\mathbf{K}_{a}, \mathbf{V}_{a}, \mathbf{W}_{a}(a=$ $1,2)$, and scalars $\varepsilon_{i} \geq 0(i=1,2)$ such that the following LMIs hold:

$$
\begin{aligned}
& {\left[\begin{array}{ll}
\overline{\boldsymbol{\Phi}} & \sqrt{h_{\delta} \mathbf{W}} \\
* & -\mathbf{Z}_{3}
\end{array}\right]<0, \quad i=1,2, \ldots, N,} \\
& {\left[\begin{array}{cc}
\overline{\boldsymbol{\Phi}} & \sqrt{h_{\delta} \mathbf{V}} \\
* & -\mathbf{Z}_{3}
\end{array}\right]<0, \quad i=1,2, \ldots, N,}
\end{aligned}
$$

where $\overline{\boldsymbol{\Phi}}=\left(\boldsymbol{\Phi}_{i, j}\right)_{7 \times 7}$ with

$$
\begin{gathered}
\overline{\boldsymbol{\Phi}}_{11}=\mathbf{P}_{12}+\mathbf{P}_{12}^{T}+\mathbf{Q}_{1}+\mathbf{Q}_{2}+\mathbf{Q}_{3}-\mathbf{Z}_{2} \\
-h_{i}^{2} \mathbf{R}_{2}-h_{\delta}^{2} \mathbf{R}_{3}+\mathbf{K}_{1} \mathbf{A}+\mathbf{A}^{T} \mathbf{K}_{1}^{T}, \\
\overline{\boldsymbol{\Phi}}_{12}=\boldsymbol{\Phi}_{12}, \quad \overline{\boldsymbol{\Phi}}_{13}=\boldsymbol{\Phi}_{13}, \quad \overline{\boldsymbol{\Phi}}_{14}=\boldsymbol{\Phi}_{14}, \\
\overline{\boldsymbol{\Phi}}_{15}=\boldsymbol{\Phi}_{15}, \quad \overline{\boldsymbol{\Phi}}_{16}=\boldsymbol{\Phi}_{16}, \quad \overline{\boldsymbol{\Phi}}_{17}=\boldsymbol{\Phi}_{17}, \\
\overline{\boldsymbol{\Phi}}_{22}=\boldsymbol{\Phi}_{22}, \quad \overline{\boldsymbol{\Phi}}_{23}=\boldsymbol{\Phi}_{23}, \quad \overline{\boldsymbol{\Phi}}_{24}=\overline{\boldsymbol{\Phi}}_{25}=0, \\
\overline{\boldsymbol{\Phi}}_{26}=\boldsymbol{\Phi}_{26}, \quad \overline{\boldsymbol{\Phi}}_{27}=\boldsymbol{\Phi}_{27},
\end{gathered}
$$

$$
\begin{aligned}
& \overline{\boldsymbol{\Phi}}_{33}=-(1-\mu) \mathbf{Q}_{1}-\mathbf{V}_{2}-\mathbf{V}_{2}^{T}+\mathbf{W}_{1}+\mathbf{W}_{1}^{T}, \\
& \overline{\boldsymbol{\Phi}}_{34}=\Phi_{34}, \quad \overline{\boldsymbol{\Phi}}_{35}=\Phi_{35}, \quad \overline{\boldsymbol{\Phi}}_{36}=\overline{\boldsymbol{\Phi}}_{37}=0, \\
& \overline{\boldsymbol{\Phi}}_{44}=\boldsymbol{\Phi}_{44}, \quad \overline{\boldsymbol{\Phi}}_{45}=0, \quad \overline{\boldsymbol{\Phi}}_{46}=\boldsymbol{\Phi}_{46}, \\
& \bar{\Phi}_{47}=\Phi_{47}, \quad \bar{\Phi}_{55}=\Phi_{55}, \quad \bar{\Phi}_{56}=\Phi_{56}, \\
& \bar{\Phi}_{57}=\Phi_{57}, \quad \bar{\Phi}_{66}=\Phi_{66}, \quad \Phi_{67}=0, \\
& \Phi_{77}=-\mathbf{R}_{3} \text {, } \\
& h_{\delta}=h_{i+1}-h_{i}=\frac{\left(h_{M}-h_{m}\right)}{N} \text {, } \\
& h_{i}=h_{1}+\frac{(i-1)\left(h_{M}-h_{m}\right)}{N} \text {, } \\
& \overline{\mathbf{K}}=\left[\begin{array}{lllllll}
\mathbf{K}_{1}^{T} & 0 & 0 & 0 & \mathbf{K}_{2}^{T} & 0 & 0
\end{array}\right]^{T}, \\
& \overline{\mathbf{V}}=\left[\begin{array}{lllllll}
0 & \mathbf{V}_{1}^{T} & \mathbf{V}_{2}^{T} & 0 & 0 & 0 & 0
\end{array}\right]^{T}, \\
& \overline{\mathbf{W}}=\left[\begin{array}{lllllll}
0 & 0 & \mathbf{W}_{1}^{T} & \mathbf{W}_{2}^{T} & 0 & 0 & 0
\end{array}\right]^{T} \text {. }
\end{aligned}
$$

\section{Numerical Examples}

Example 1. Consider the following neutral time-delay system with

$$
\mathbf{A}=\left[\begin{array}{ll}
-1.2 & 0.1 \\
-0.1 & -1
\end{array}\right], \quad \mathbf{B}=\left[\begin{array}{cc}
-0.6 & 0.7 \\
-1 & -0.8
\end{array}\right]
$$

For given values of $\alpha, \beta$, and $\mu$, we apply Theorem 4 to calculate the maximal allowable value $h_{M}$ that guarantees that the asymptotical stability of the system is listed in Table 1. From Table 1, it is easy to see that our proposed stability criterion gives much less conservative results than those in $[21,22]$.

Example 2. Consider the system (27) with the following matrices:

$$
\mathbf{A}=\left[\begin{array}{cc}
0 & 1 \\
-1 & -2
\end{array}\right], \quad \mathbf{B}=\left[\begin{array}{cc}
0 & 0 \\
-1 & 1
\end{array}\right]
$$

For given $h_{m}$ and $\mu$, we calculate the allowable upper bound of $h_{M}$ that guarantees the asymptotical stability of system (27). Using different methods, computational results are obtained and these are listed in Table 2. From Table 2, it can be seen that our results are less conservative than the existing criteria.

\section{Conclusion}

This paper studies the problem of robust delay-dependent stability for a class of linear systems with interval timevarying delay and nonlinear perturbations. Based on the delay-partitioning approach, the less conservative delaydependent stability conditions are derived. The reduction in 
TABLE 1: Admissible upper bounds $h_{2}$ for various $\mu$ and $h_{m}=0,0.5,1$.

\begin{tabular}{|c|c|c|c|c|c|c|c|}
\hline \multirow{2}{*}{$h_{m}$} & \multirow{2}{*}{$\begin{array}{c}\alpha, \beta \\
\mu\end{array}$} & \multicolumn{3}{|c|}{$\alpha=0, \beta=0.1$} & \multicolumn{3}{|c|}{$\alpha=0.1, \beta=0.1$} \\
\hline & & 0.5 & 0.9 & 1.1 & 0.5 & 0.9 & 1.1 \\
\hline \multirow{4}{*}{0} & {$[21]$} & 1.442 & 1.280 & 1.280 & 1.284 & 1.209 & 1.209 \\
\hline & {$[22]$} & 1.443 & 1.408 & 1.408 & 1.287 & 1.279 & 1.279 \\
\hline & Theorem $4(N=2)$ & 1.6299 & 1.4938 & 1.4938 & 1.4743 & 1.3943 & 1.3943 \\
\hline & Theorem $4(N=3)$ & 1.6866 & 1.6866 & 1.6866 & 1.5304 & 1.5304 & 1.5304 \\
\hline \multirow{4}{*}{0.5} & {$[21]$} & 1.442 & 1.338 & 1.338 & 1.284 & 1.245 & 1.245 \\
\hline & {$[22]$} & 1.558 & 1.558 & 1.558 & 1.384 & 1.384 & 1.384 \\
\hline & Theorem $4(N=2)$ & 1.6618 & 1.6618 & 1.6618 & 1.5272 & 1.5272 & 1.5272 \\
\hline & Theorem $4(N=3)$ & 1.8599 & 1.8599 & 1.8599 & 1.6622 & 1.6622 & 1.6622 \\
\hline \multirow{4}{*}{1} & {$[21]$} & 1.543 & 1.543 & 1.543 & 1.408 & 1.408 & 1.408 \\
\hline & {$[22]$} & 1.760 & 1.760 & 1.760 & 1.532 & 1.532 & 1.532 \\
\hline & Theorem $4(N=2)$ & 1.8824 & 1.8824 & 1.8824 & 1.7059 & 1.7059 & 1.7059 \\
\hline & Theorem $4(N=3)$ & 2.0650 & 2.0650 & 2.0650 & 1.8188 & 1.8188 & 1.8188 \\
\hline
\end{tabular}

TABLE 2: Maximum bounds $h_{M}$ for a given $h_{m}$.

\begin{tabular}{ccccccc}
\hline$\mu$ & Method & $h_{m}=1$ & $h_{m}=2$ & $h_{m}=3$ & $h_{m}=4$ & $h_{m}=5$ \\
\hline \multirow{4}{*}{0.3} & 2.2125 & 2.4091 & 3.3342 & 5.2799 & 5.2393 \\
& {$[4]$} & 2.2474 & 2.4798 & 3.3896 & 4.3250 & 5.2773 \\
& {$[8]$} & 2.3564 & 3.0484 & 3.8779 & 5.6475 \\
& {$[18](N=2)$} & 2.5378 & 3.0804 & 3.9171 & 4.7933 & 5.6967 \\
& Corollary 7 $(N=2)$ & 2.7077 & 3.4408 & 4.2307 & 5.0463 & 5.9313 \\
& {$[18](N=3)$} & 2.7469 & 3.4900 & 4.2899 & 6.0030 \\
\hline
\end{tabular}

the conservatism of the proposed stability criteria is mainly attributed to the new $\mathrm{L}-\mathrm{K}$ functional which contains some triple-integral terms and augment terms for each divided segment. Numerical examples have illustrated the effectiveness of the proposed method.

\section{Conflict of Interests}

The authors declare that there is no conflict of interests regarding the publication of this paper.

\section{Acknowledgment}

The research is supported by the National Natural Science Foundation of China, Grant nos. 61304001 and 61304103.

\section{References}

[1] L. Wu and W. X. Zheng, "Passivity-based sliding mode control of uncertain singular time-delay systems," Automatica, vol. 45, no. 9, pp. 2120-2127, 2009.

[2] W. Chen and L. Qiu, "Stabilization of networked control systems with multirate sampling," Automatica, vol. 49, no. 6, pp. 15281537, 2013.

[3] Y. He, M. Wu, J.-H. She, and G.-P. Liu, "Parameter-dependent Lyapunov functional for stability of time-delay systems with polytopic-type uncertainties," IEEE Transactions on Automatic Control, vol. 49, no. 5, pp. 828-832, 2004.
[4] Y. He, Q.-G. Wang, L. Xie, and C. Lin, "Further improvement of free-weighting matrices technique for systems with timevarying delay," IEEE Transactions on Automatic Control, vol. 52, no. 2, pp. 293-299, 2007.

[5] M. Wu, Y. He, J.-H. She, and G.-P. Liu, "Delay-dependent criteria for robust stability of time-varying delay systems," Automatica, vol. 40, no. 8, pp. 1435-1439, 2004.

[6] P. Park and J. W. Ko, "Stability and robust stability for systems with a time-varying delay," Automatica, vol. 43, no. 10, pp. 1855$1858,2007$.

[7] L. Wu and W. X. Zheng, "Weighted $H_{\infty}$ model reduction for linear switched systems with time-varying delay," Automatica, vol. 45, no. 1, pp. 186-193, 2009.

[8] H. Shao, "New delay-dependent stability criteria for systems with interval delay," Automatica, vol. 45, no. 3, pp. 744-749, 2009.

[9] X.-M. Zhang and Q.-L. Han, "New Lyapunov-Krasovskii functionals for global asymptotic stability of delayed neural networks," IEEE Transactions on Neural Networks, vol. 20, no. 3, pp. 533-539, 2009.

[10] O. M. Kwon, J. H. Park, and S. M. Lee, "An improved delay-dependent criterion for asymptotic stability of uncertain dynamic systems with time-varying delays," Journal of Optimization Theory and Applications, vol. 145, no. 2, pp. 343-353, 2010.

[11] K. Ramakrishnan and G. Ray, "Robust stability criteria for uncertain neutral systems with interval time-varying delay," Journal of Optimization Theory and Applications, vol. 149, no. 2, pp. 366-384, 2011.

[12] L. Wu, X. Yang, and H.-K. Lam, "Dissipativity analysis and sysnthesis systems for discrete-time T-S fuzzy stochastic systems 
with time-varyng delay," IEEE Transactions on Fuzzy Systems, vol. 22, no. 2, pp. 380-394, 2014.

[13] R. Rakkiyappan, P. Balasubramaniam, and R. Krishnasamy, "Delay dependent stability analysis of neutral systems with mixed time-varying delays and nonlinear perturbations," Journal of Computational and Applied Mathematics, vol. 235, no. 8, pp. 2147-2156, 2011.

[14] Q.-L. Han, "Improved stability criteria and controller design for linear neutral systems," Automatica, vol. 45, no. 8, pp. 1948-1952, 2009.

[15] Q.-L. Han, "A discrete delay decomposition approach to stability of linear retarded and neutral systems," Automatica, vol. 45, no. 2, pp. 517-524, 2009.

[16] C. Peng and Y.-C. Tian, "Improved delay-dependent robust stability criteria for uncertain systems with interval timevarying delay," IET Control Theory \& Applications, vol. 2, no. 9, pp. 752-761, 2008.

[17] K. Ramakrishnan and G. Ray, "Robust stability criteria for uncertain linear systems with interval time-varying delay," Journal of Control Theory and Applications, vol. 9, no. 4, pp. 559566, 2011.

[18] C. Wang and Y. Shen, "Delay partitioning approach to robust stability analysis for uncertain stochastic systems with interval time-varying delay," IET Control Theory \& Applications, vol. 6, no. 7, pp. 875-883, 2012.

[19] Q.-L. Han, "Robust stability for a class of linear systems with time-varying delay and nonlinear perturbations," Computers \& Mathematics with Applications, vol. 47, no. 8-9, pp. 1201-1209, 2004.

[20] Z. Zuo and Y. Wang, "New stability criterion for a class of linear systems with time-varying delay and nonlinear perturbations," IEE Proceedings. Control Theory \& Applications, vol. 153, no. 5, pp. 623-626, 2006.

[21] W. Zhang, X.-S. Cai, and Z.-Z. Han, "Robust stability criteria for systems with interval time-varying delay and nonlinear perturbations," Journal of Computational and Applied Mathematics, vol. 234, no. 1, pp. 174-180, 2010.

[22] K. Ramakrishnan and G. Ray, "Delay-range-dependent stability criterion for interval time-delay systems with nonlinear perturbations," International Journal of Automation and Computing, vol. 8, no. 1, pp. 141-146, 2011.

[23] P. Balasubramaniam and G. Nagamani, "A delay decomposition approach to delay-dependent passivity analysis for interval neural networks with time-varying delay," Neurocomputing, vol. 74, no. 10, pp. 1646-1653, 2011.

[24] L. Wu, X. Su, and P. Shi, "Sliding mode control with bounded $L_{2}$ gain performance of Markovian jump singular time-delay systems," Automatica, vol. 48, no. 8, pp. 1929-1933, 2012.

[25] D. Yue, E. Tian, and Y. Zhang, "A piecewise analysis method to stability analysis of linear continuous/discrete systems with time-varying delay," International Journal of Robust and Nonlinear Control, vol. 19, no. 13, pp. 1493-1518, 2009. 


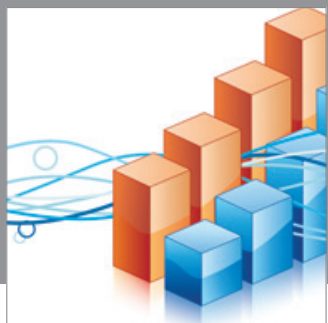

Advances in

Operations Research

mansans

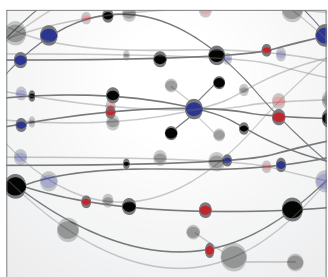

The Scientific World Journal
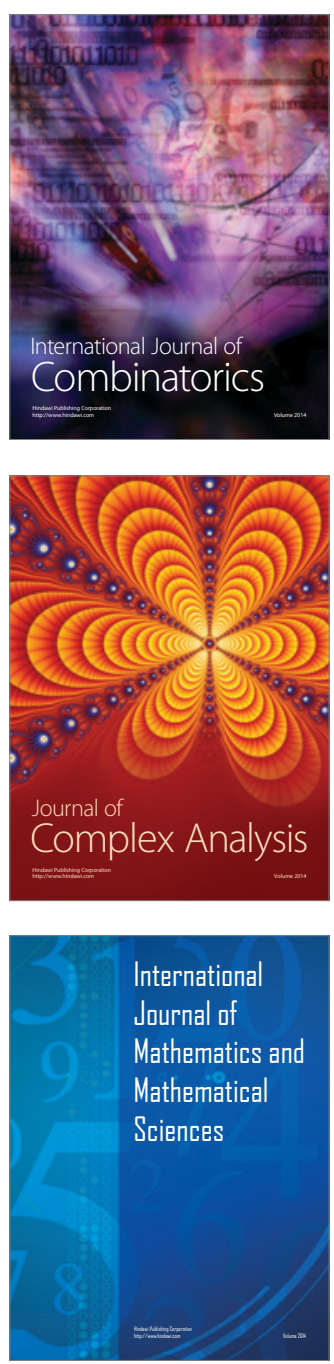
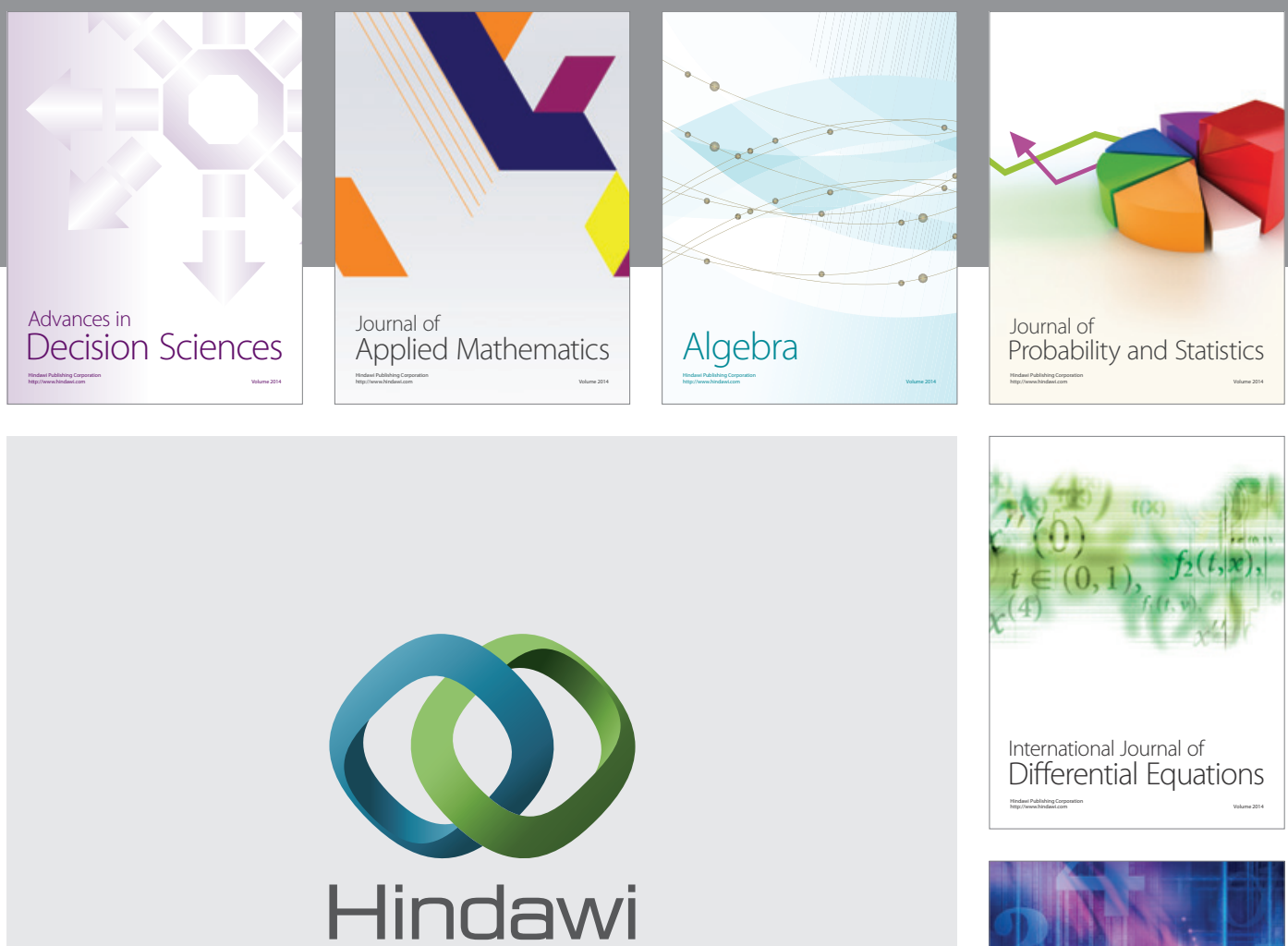

Submit your manuscripts at http://www.hindawi.com
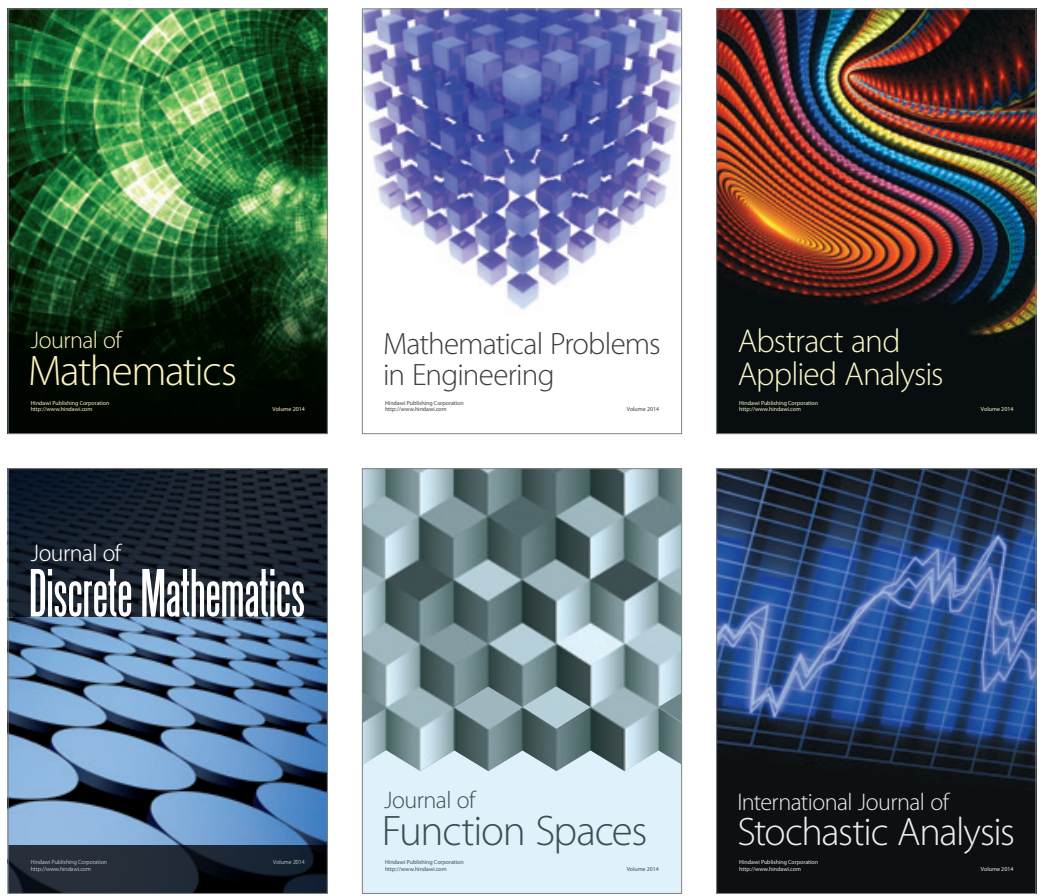

Journal of

Function Spaces

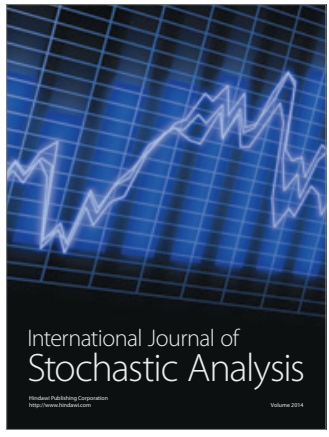

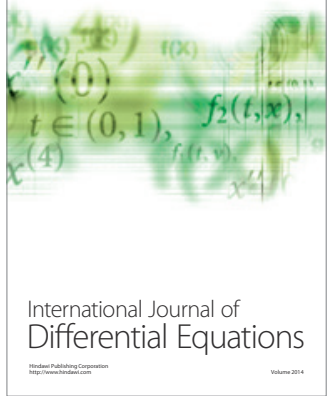
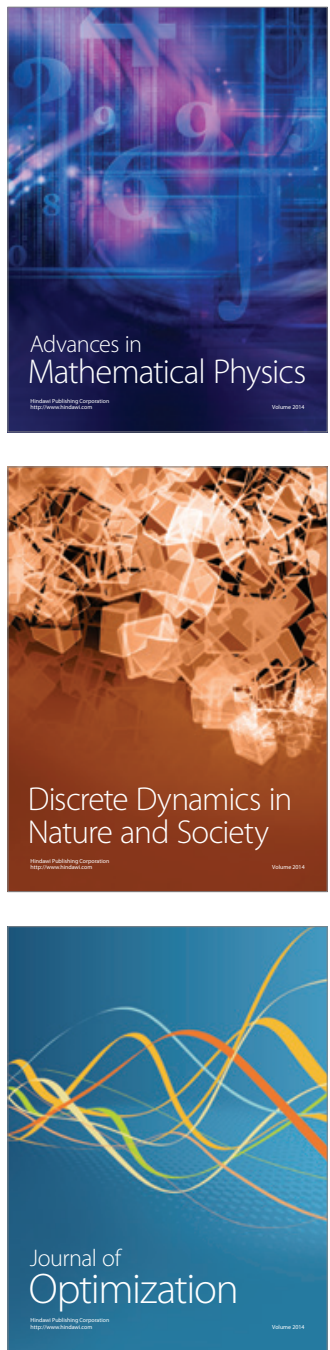